\title{
A LEMMA ON THE GALTON-WATSON PROCESS AND SOME OF ITS CONSEQUENCES
}

\section{F. PAPANGELOU ${ }^{1}$}

Many of the known limit theorems on the Galton-Watson process are immediate corollaries of the elementary lemma given below. This lemma enables us to calculate easily limits of various types of conditional distributions, special cases of which had earlier been tackled by diverse and more complicated methods.

Let $F(x)=\sum_{j=0}^{\infty} p_{1} x^{j}$ be the distribution generating function of the offspring of one individual in a Galton-Watson process. We assume $0<p_{10}<1$. Throughout the present note $r$ will denote the least positive integer such that $p_{1 r}>0$. We set $F_{0}(x)=x, F_{n+1}(x)$ $=F\left(F_{n}(x)\right)$ and denote by $q$ the probability of extinction of the progeny of one individual. Then $q=1$ or $<1$ according as $m=\sum_{j=1}^{\infty} j p_{1 j}$ is $\leqq 1$ or $>1$. Further $F_{n}(x) \uparrow q$ if $0 \leqq x<q, F_{n}(x) \downarrow q$ if $q<x<1$ while $F(q)=q, F(1)=1$, [3]. The terms "increasing" and "decreasing" as used below do not mean strict monotonicity.

It is well known that $F_{n}(x)^{i}=\sum_{j=0}^{\infty} p_{i j}^{(n)} x^{j}$ so that

$$
p_{i j}^{(n)}=\frac{1}{j !} \frac{d^{j}}{d x^{j}}\left[F_{n}(x)^{i}\right]_{x=0}
$$

Further

$$
F_{n}^{\prime}(0)=F_{n}^{\prime \prime}(0)=\cdots=F_{n}^{(r-1)}(0)=0 \quad \text { for any } n
$$

and

$$
F_{n}^{(r)}(0)=F^{\prime}\left(F_{n-1}(0)\right) F_{n-1}^{(r)}(0)>0 .
$$

It is easy to prove by induction on $j$ that for every $n \geqq 1$ and every $j \geqq 1$

$$
F_{n}^{(j)}(x)=A_{n, j}(x)+F^{\prime}\left(F_{n-1}(x)\right) F_{n-1}^{(j)}(x), \quad-1<x<1,
$$

where $A_{n, j}(x)$ is a power series in $x$ with nonnegative coefficients.

Lemma. For fixed $j \geqq 1$ the sequence $p_{1 j}^{(n)} / p_{1 r}^{(n)}, n=1,2, \ldots$ is increasing.

Received by the editors July 17, 1967.

1 The present paper was written while the author was holding a research fellowship of the Alexander von Humboldt Foundation (Germany). 
Proof. By (2) and (3)

$$
\begin{aligned}
\frac{p_{1 j}^{(n+1)}}{p_{1 r}^{(n+1)}} & =\frac{\frac{1}{j !} F_{n+1}^{(j)}(0)}{\frac{1}{r !} F_{n+1}^{(r)}(0)}=\frac{\frac{1}{j !}\left[A_{n+1, j}(0)+F^{\prime}\left(F_{n}(0)\right) F_{n}^{(j)}(0)\right]}{\left[\frac{1}{r !} F^{\prime}\left(F_{n}(0)\right) F_{n}^{(r)}(0)\right]} \\
& \geqq \frac{\frac{1}{j !} F_{n}^{(j)}(0)}{\left[\frac{1}{r !} F_{n}^{(r)}(0)\right]}=\frac{p_{1 j}^{(n)}}{p_{1 r}^{(n)}} .
\end{aligned}
$$

Let $\pi(j)=\lim _{n \rightarrow \infty} p_{1 j}^{(n)} / p_{1 r}^{(n)}(j \geqq 1)$ and note that the sequence

$$
p_{1 r}^{(n+1)} / p_{1 r}^{(n)}=\frac{1}{r !} F^{\prime}\left(F_{n}(0)\right) F_{n}^{(r)}(0) /\left[\frac{1}{r !} F_{n}^{(r)}(0)\right]=F^{\prime}\left(F_{n}(0)\right)
$$

is increasing and converges to $F^{\prime}(q)$. We set $\gamma=F^{\prime}(q)$. Then $\gamma<1$ if and only if $m \neq 1$; if $m \leqq 1$ then $\gamma=m$.

The following theorem can be pieced together from results either explicitly or implicitly contained in the union $[4] \cup[5] \cup[6] \cup[7]$ $\cup[10] \cup[11]$, but the methods of these authors are sometimes sophisticated, and each is applicable to only one of the three cases $m \gtreqless 1$. The lemma provides the basis for a simple unified proof.

Theorem. $\pi(j)<+\infty$ for all $j$. For any $i \geqq 1, j \geqq 1$

$$
\lim _{n \rightarrow \infty} p_{i j}^{(n+k)} / p_{1 r}^{(n)}=\gamma^{k} i q^{i-1} \pi(j) \quad(k=0, \pm 1, \pm 2, \cdots)
$$

and

$$
\gamma \pi(j)=\sum_{i=1}^{\infty} \pi(i) p_{i j}, \quad j \geqq 1 .
$$

$\{\pi(j)\}$ is the unique (to within a constant factor) nonnegative solution of (5). The generating function $A(x)=\sum_{j=1}^{\infty} \pi(j) x^{j}$ is finite in $(-1,1)$ and $A(1)<+\infty$ or $=+\infty$ according as $m<1$ or $m \geqq 1$. Further

$$
A(F(x))=\gamma A(x)+A(F(0))
$$

for every $x \in(-1,1)$ and $A(x)$ is the unique (to within a constant factor) solution of $(6)$ in the interval $[0, q)$ which has a nonnegative and increasing derivative. 
Proof.

$$
p_{i j}^{(n)}=\sum_{\left(h_{1}, h_{8}, \cdots, h_{i}\right) ; 0 \leqq h_{\nu} \leq j ; h_{1}+h_{2}+\cdots+h_{i}=j} p_{1 h_{1}}^{(n)} p_{1 h_{2}}^{(n)} \cdots p_{1 h_{i}}^{(n)} .
$$

Separating those terms having some $h_{\nu}$ equal to $j$ we get

$$
\frac{p_{i j}^{(n)}}{p_{1 r}^{(n)}}=i\left(p_{10}^{(n)}\right)^{i-1} \frac{p_{1 j}^{(n)}}{p_{1 r}^{(n)}}+\sum_{\left(h_{1}, h_{2}, \cdots, h_{i}\right) ; 0 \leqslant h_{j}<j ; h_{1}+h_{2}+\cdots+h_{i}=j} \frac{p_{1 h_{1}}^{(n)} \cdots p_{1 h_{i}}^{(n)}}{p_{1 r}^{(n)}}
$$

The limit of the first term on the right is $i q^{i-1} \pi(j)$ while the limit of the second term is 0 because each summand has at least two $h_{p}$ 's greater than 0 , say $h_{s}, h_{t}$, and hence

$$
p_{1 h_{1}}^{(n)} \cdots p_{1 h_{i}}^{(n)} / p_{1 r}^{(n)} \leqq\left[p_{1 h_{s}}^{(n)} / p_{1 r}^{(n)}\right] \cdot p_{1 h_{t}}^{(n)} \rightarrow \pi\left(h_{8}\right) \cdot 0 .
$$

Now (5) follows from the lemma and

$$
\frac{p_{1 r}^{(n+1)}}{p_{1 r}^{(n)}} \cdot \frac{p_{1 j}^{(n+1)}}{p_{1 r}^{(n+1)}}=\sum_{i=1}^{\infty} \frac{p_{1 i}^{(n)}}{p_{1 r}^{(n)}} p_{i j}
$$

If $0<x<q$ then there is an $n$ such that $x<F_{n}(0)=p_{10}^{(n)}<q$ and iteration of (5) yields

$$
\gamma^{n} \pi(r)=\sum_{i=1}^{\infty} \pi(i) p_{i r}^{(n)} \geqq \sum_{i=1}^{\infty} \pi(i) i\left(p_{10}^{(n)}\right)^{i-1} p_{1 r}^{(n)} \geqq p_{1 r}^{(n)} \sum_{i=1}^{\infty} \pi(i) x^{i} .
$$

Hence $\pi(i)<+\infty$ for every $i$ and $A(x)$ is finite in $(-q, q)$. It is more difficult to show that if $q<1$ (i.e. if $m>1)$ then $A(x)$ is finite in $(q, 1)$. Let $q<x<1$ and recall that $F_{n}(x) \downarrow q$. By the mean-value theorem and (2)

$$
\begin{aligned}
\sum_{j=1}^{\infty} \pi(j) x^{j} & =\lim _{n \rightarrow \infty} \sum_{j=1}^{\infty} \frac{p_{1 j}^{(n)}}{p_{1 r}^{(n)}} x^{j}=\lim _{n \rightarrow \infty} \frac{F_{n}(x)-F_{n}(0)}{\frac{1}{r !} F_{n}^{(r)}(0)} \leqq \lim _{n \rightarrow \infty} \frac{F_{n}^{\prime}(x) \cdot x}{F_{(n)}^{(r)}(0) / r !} \\
& =(r !) x \lim _{n \rightarrow \infty} \frac{F^{\prime}(x) F^{\prime}(F(x)) \cdots F^{\prime}\left(F_{n-1}(x)\right)}{F^{(r)}(0) F^{\prime}(F(0)) \cdots F^{\prime}\left(F_{n-1}(0)\right)}
\end{aligned}
$$

so that, by the well-known criterion for infinite products, it is sufficient to prove $\sum_{n=1}^{\infty}\left(\left[F^{\prime}\left(F_{n}(x)\right) / F^{\prime}\left(F_{n}(0)\right)\right]-1\right)<+\infty$ or equivalently $\sum_{n=1}^{\infty}\left\{F^{\prime}\left(F_{n}(x)\right)-F^{\prime}\left(F_{n}(0)\right)\right\}<+\infty$. A pplying repeatedly the meanvalue theorem we obtain 


$$
\begin{aligned}
& F^{\prime}\left(F_{n}(x)\right)-F^{\prime}\left(F_{n}(0)\right) \\
& \leqq F^{\prime \prime}\left(F_{n}(x)\right)\left(F_{n}(x)-F_{n}(0)\right) \\
& \leqq F^{\prime \prime}\left(F_{n}(x)\right) F_{n-1}^{\prime}(F(x))(F(x)-F(0)) \\
&=F^{\prime \prime}\left(F_{n}(x)\right) F_{n-1}^{\prime}(F(x))(x-F(x)) \cdot[F(x)-F(0)] /[x-F(x)] \\
& \leqq F^{\prime \prime}\left(F_{n}(x)\right)\left(F_{n-1}(x)-F_{n}(x)\right) \cdot[F(x)-F(0)] /[x-F(x)] \\
& \leqq\left\{F^{\prime}\left(F_{n-1}(x)\right)-F^{\prime}\left(F_{n}(x)\right)\right\} \cdot[F(x)-F(0)] /[x-F(x)]
\end{aligned}
$$

and clearly $\sum_{n=1}^{\infty}\left\{F^{\prime}\left(F_{n-1}(x)\right)-F^{\prime}\left(F_{n}(x)\right)\right\}=F^{\prime}(x)-F^{\prime}(q)$.

Multiplying (5) by $x^{j}$ and summing over $j$ we find

$$
\gamma A(x)=\sum_{i=1}^{\infty} \pi(i)\left(F(x)^{i}-F(0)^{i}\right)=A(F(x))-A(F(0))
$$

so that $(6)$ holds in $(-1,1)$. This implies

$$
\begin{aligned}
A\left(F_{n}(x)\right)=\gamma^{n} A(x)+A(F(0))\left[1+\gamma+\cdots+\gamma^{n-1}\right] & , \\
-1 & <x<1
\end{aligned}
$$

hence

$$
A^{\prime}\left(F_{n}(x)\right) F_{n}^{\prime}(x)=\gamma^{n} A^{\prime}(x)
$$

and

$$
\lim _{n \rightarrow \infty} \frac{A^{\prime}\left(F_{n+1}(x)\right)}{A^{\prime}\left(F_{n}(x)\right)}=\lim _{n \rightarrow \infty} \gamma \frac{F_{n}^{\prime}(x)}{F_{n+1}^{\prime}(x)}=\lim _{n \rightarrow \infty} \gamma \frac{F_{n}^{\prime}(x)}{F^{\prime}\left(F_{n}(x)\right) F_{n}^{\prime}(x)}=1
$$

when $0 \leqq x<1$. (For $x=0$ we write $F_{n}(0)=F_{n-1}\left(x_{0}\right)$ where $x_{0}=F(0)$, because $F_{n}^{\prime}(0)=0, A^{\prime}(0)=0$ if $r>1$.)

Let $B(x)$ be another solution of $(6)$ in $[0, q)$ as described in the theorem. Then

$$
B^{\prime}\left(F_{n}(x)\right) / A^{\prime}\left(F_{n}(x)\right)=B^{\prime}(x) / A^{\prime}(x), \quad 0<x<q .
$$

Set

$$
c=B^{\prime}(F(0)) / A^{\prime}(F(0))=B^{\prime}\left(F_{n}(0)\right) / A^{\prime}\left(F_{n}(0)\right), \quad n=1,2, \cdots .
$$

If $x$ is any point in $[0, q)$ there is $\nu \geqq 0$ such that $F_{\nu}(0) \leqq x<F_{\nu+1}(0)$ so that by (9)

$$
\frac{B^{\prime}(x)}{A^{\prime}(x)}=\frac{B^{\prime}\left(F_{n}(x)\right)}{A^{\prime}\left(F_{n}(x)\right)} \leqq \frac{B^{\prime}\left(F_{v+n+1}(0)\right)}{A^{\prime}\left(F_{\nu+n}(0)\right)}=c \cdot \frac{A^{\prime}\left(F_{\nu+n+1}(0)\right)}{A^{\prime}\left(F_{\nu+n}(0)\right)} \rightarrow c
$$

(when $n \rightarrow+\infty$ ) and similarly $B^{\prime}(x) / A^{\prime}(x) \geqq c$. Thus $B^{\prime}(x) / A^{\prime}(x)=c$, $B(x)=c A(x)+c^{\prime}$. Since $B(x)$ satisfies $(6) B(0)=0, c^{\prime}=0$. 
The uniqueness of $\{\pi(j)\}$ in (5) follows from the fact that if $\{\alpha(j)\}$ is any nonnegative solution then $\sum_{j=1}^{\infty} \alpha(j) x^{j}$ is finite in $(-q, q)$ (as before) and a solution of (6) in $[0, q)$. Finally by (7) $A(1)$ is finite if $m<1$ and infinite if $m=1$. In the case $m>1$

$$
A(1)=\sum_{j=1}^{\infty} \pi(j)=\lim _{n \rightarrow \infty} \sum_{j=1}^{\infty} \frac{p_{1 j}^{(n)}}{p_{1 r}^{(n)}}=\lim _{n \rightarrow \infty} \frac{1-F_{n}(0)}{p_{1 r}^{(n)}}=+\infty
$$

because $1-F_{n}(0) \rightarrow 1-q$ while $p_{1 r}^{(n)} \rightarrow 0$. This completes the proof.

We shall call the process $R$-positive [12] if the sequence

$$
\frac{p_{1 r}^{(n)}}{\gamma^{n}}=\frac{1}{r !} F^{(r)}(0) F^{\prime}(F(0)) F^{\prime}\left(F_{2}(0)\right) \cdots F^{\prime}\left(F_{n-1}(0)\right) \frac{1}{\gamma^{n}}
$$

(which is decreasing) has a positive limit. Clearly

$$
\begin{aligned}
\frac{p_{1 r}^{(n)}}{\gamma^{n}} & =\frac{p_{1 r}^{(n)}}{F^{\prime}(q)^{n}}=\frac{p_{1 r}^{(n)}}{F_{n}{ }^{\prime}(q)} \\
& =\left(\sum_{j=1}^{\infty} j \frac{p_{1 j}^{(n)}}{p_{1 r}^{(n)}} q^{j-1}\right)^{-1} \downarrow\left(\sum_{j=1}^{\infty} j \pi(j) q^{j-1}\right)^{-1}=\frac{1}{A^{\prime}(q)}
\end{aligned}
$$

so that we have $R$-positivity if and only if $A^{\prime}(q)<+\infty$.

Corollary 1 [11]. If $m>1$ then the process is $R$-positive.

When $m<1$ the necessary and sufficient condition for $R$-positivity was shown in [11] to be

$$
\sum_{j=1}^{\infty} j(\log j) p_{1 j}<+\infty .
$$

If $m<1$ the necessary and sufficient condition for $A^{\prime \prime}(1)<+\infty$ is $F^{\prime \prime}(1)<+\infty$; this follows from the equality

$$
\sum_{j=1}^{\infty} j(j-1) \frac{p_{1 j}^{(n)}}{p_{1 r}^{(n)}}=\frac{F_{n}^{\prime \prime}(1)}{p_{1 r}^{(n)}}=F^{\prime \prime}(1) \frac{\gamma^{n}}{p_{1 r}^{(n)}} \cdot \frac{1-\gamma^{n}}{\gamma(1-\gamma)} .
$$

CoROllaRy 2 . For $0 \leqq x \leqq q$ and $i \geqq 1$ the sequences $\sum_{j=1}^{\infty} p_{i j}^{(n)} x^{j} / p_{1 r}^{(n)}$, $\sum_{j=1}^{\infty} j p_{i j}^{(n)} x^{j} / p_{1 r}^{(n)}, n=1,2, \cdots$ are increasing and

$$
\lim _{n \rightarrow \infty} \sum_{j=1}^{\infty} \frac{p_{i j}^{(n)}}{p_{1 r}^{(n)}} x^{j}=i q^{i-1} \sum_{j=1}^{\infty} \pi(j) x^{j}
$$




$$
\lim _{n \rightarrow \infty} \sum_{j=1}^{\infty} j \frac{p_{i j}^{(n)}}{p_{1 r}^{(n)}} x^{j}=i q^{i-1} \sum_{j=1}^{\infty} j \pi(j) x^{j} .
$$

If $q<1,(13)$ and (14) hold for every $x \in[0,1)$.

In fact

$$
\begin{aligned}
\sum_{j=1}^{\infty} p_{i j}^{(n)} x^{j}= & F_{n}(x)^{i}-F_{n}(0)^{i}=\left(F_{n}(x)-F_{n}(0)\right) \\
& \cdot\left(F_{n}(x)^{i-1}+\cdots+F_{n}(0)^{i-1}\right) \\
= & \left(\sum_{j=1}^{\infty} p_{1 j}^{(n)} x^{j}\right)\left(F_{n}(x)^{i-1}+\cdots+F_{n}(0)^{i-1}\right) .
\end{aligned}
$$

Similarly

$$
\sum_{j=1}^{\infty} j p_{i j}^{(n)} x^{j}=x \frac{d}{d x}\left\{F_{n}(x)^{i}\right\}=x i F_{n}(x)^{i-1} F_{n}^{\prime}(x)=i F_{n}(x)^{i-1} \sum_{j=1}^{\infty} j p_{1 j}^{(n)} x^{j} .
$$

We now illustrate the use of the lemma and Corollary 2 in the derivation of limit theorems. Let $Z_{0}, Z_{1}, Z_{2}, \cdots$ be a Galton-Watson process with generating function $F(x)$ and denote by $T$ the time to extinction $\left(T=+\infty\right.$ in case of survival). If $P\left[Z_{0}=1\right]=1$ then the results below follow directly from the lemma. We prefer to base the proofs on Corollary 2 and the continuity theorem [2, p. 262] and thus widen the scope of these propositions by allowing $P\left[Z_{0}=i\right]=\lambda_{i}$ where $\sum_{i=1}^{\infty} \lambda_{i}=1$. We introduce

Assumption A. Either $m \leqq 1$ and $\sum_{i=1}^{\infty} i \lambda_{i}<+\infty$ or $m>1$ and $\sum_{i=1}^{\infty} i \lambda_{i}$ is arbitrary and prove:

(I). Let $k \geqq 0$ be fixed. Under Assumption A

$$
\begin{aligned}
\lim _{n \rightarrow \infty} P\left[Z_{n}=\right. & j \mid n+k<T<+\infty] \\
& =\pi(j) \sum_{h=1}^{\infty} p_{j h}^{(k)} q^{h} /\left(\gamma^{k} \sum_{h=1}^{\infty} \pi(h) q^{h}\right)
\end{aligned}
$$

which is zero if $m=1$ and a probability distribution on $\{j\}$ if $m \neq 1$.

Proof. Note that $\sum_{h=1}^{\infty} p_{j h}^{(\boldsymbol{k})} q^{h}=F_{k}(q)^{j}-p_{j 10}^{(\mathbf{k})}=q^{j}-\left(p_{10}^{(\mathbf{k})}\right)^{j}$ so that

$$
\begin{aligned}
P\left[Z_{n}=j \mid n+\right. & k<T<+\infty] \\
& =\sum_{i=1}^{\infty} \lambda_{i} p_{i j}^{(n)}\left[q^{j}-\left(p_{10}^{(k)}\right)^{j}\right] / \sum_{h=1}^{\infty} \sum_{i=1}^{\infty} \lambda_{i} p_{i h}^{(n+k)} q^{h} .
\end{aligned}
$$

The corresponding generating function is easily seen to be 


$$
\frac{\sum_{i=1}^{\infty} \lambda_{i}\left[\sum_{j=1}^{\infty} \frac{p_{i j}^{(n)}}{p_{1 r}^{(n)}}(q x)^{j}\right]-\sum_{i=1}^{\infty} \lambda_{i}\left[\sum_{j=1}^{\infty} \frac{p_{i j}^{(n)}}{p_{1 r}^{(n)}}\left(p_{10}^{(k)} x\right)^{j}\right]}{\frac{p_{1 r}^{(n+k)}}{p_{1 r}^{(n)}} \cdot \sum_{i=1}^{\infty} \lambda_{i} \sum_{h=1}^{\infty} \frac{p_{i h}^{(n+k)}}{p_{1 r}^{(n+k)}} q^{h}}
$$

which by Corollary 2 and Assumption A converges to

$$
\frac{\sum_{i=1}^{\infty} \lambda_{i} i q^{i-1} \sum_{j=1}^{\infty} \pi(j)\left[q^{j}-\left(p_{10}^{(k)}\right)^{j}\right] x^{j}}{\gamma^{k} \sum_{i=1}^{\infty} \lambda_{i} i q^{i-1} \sum_{h=1}^{\infty} \pi(h) q^{h}}=\sum_{j=1}^{\infty} \frac{\pi(j)\left(q^{j}-\left(p_{10}^{(k)}\right)^{j}\right)}{\gamma^{k} \sum_{h=1}^{\infty} \pi(h) q^{h}} x^{j} .
$$

That (15) is a probability distribution when $m \neq 1$ follows from (5).

The special case $m<1, k=0, \lambda_{1}=1$ is the well-known theorem of Yaglom [13], proved originally under the assumption $F^{\prime \prime}(1)<+\infty$ and later extended to the case $F^{\prime \prime}(1)=+\infty$ in [4] and [5] independently. The case $m>1, k=0$ is due to E. Seneta and D. Vere-Jones who proved a general result on $R$-positive chains [11, Theorem 4.2, p. 417].

(II). Under Assumption A the conditional mean

$$
\sum_{j=1}^{\infty} j P\left[Z_{n}=j \mid n+k<T<+\infty\right]
$$

converges to $+\infty$ if $m=1$ and to

$$
\sum_{j=1}^{\infty} j \pi(j) \sum_{h=1}^{\infty} p_{j h}^{(k)} q^{h} /\left(\gamma^{k} \sum_{h=1}^{\infty} \pi(h) q^{h}\right)=\frac{A^{\prime}(q)-A^{\prime}\left(p_{10}^{(k)}\right)}{\gamma^{k} A(q)}
$$

if $m \neq 1$. Thus the limit is finite if and only if the process is $R$-positive.

Proof. If $m \neq 1$ we work as in (I), using (14). If $m=1(q=1)$ then for every $J$

$$
\begin{aligned}
\sum_{j=1}^{\infty} j P\left[Z_{n}=j \mid n+k<T<+\infty\right] \\
\geqq J \frac{\sum_{i=1}^{\infty} \lambda_{i} \sum_{j=J}^{\infty} \frac{p_{i j}^{(n)}}{p_{1 r}^{(n)}}-\sum_{i=1}^{\infty} \lambda_{i} \sum_{j=J}^{\infty} \frac{p_{i j}^{(n)}}{p_{1 r}^{(n)}}\left(p_{10}^{(k)}\right)^{j}}{\frac{p_{1 r}^{(n+k)}}{p_{1 r}^{(n)}} \cdot \sum_{i=1}^{\infty} \lambda_{i} \sum_{h=1}^{\infty} \frac{p_{i h}^{(n+k)}}{p_{1 r}^{(n+k)}}}
\end{aligned}
$$

and the result follows from the fact that $\sum_{j=1}^{\infty} \pi(j)=+\infty$. The case 
$m<1, \quad k=0, \quad \lambda_{1}=1 \quad$ yields $m^{n} /\left(1-F_{n}(0)\right) \rightarrow \sum_{j=1}^{\infty} j \pi(j) / \sum_{j=1}^{\infty} \pi(j)$ [8], [4].

(III). Under Assumption A if $k \geqq 1$ is fixed

(17) $\lim _{n \rightarrow \infty} P\left[Z_{n}=j \mid T=n+k\right]=\pi(j) \sum_{h=1}^{\infty} p_{j h}^{(k-1)} p_{h 0} / \gamma^{k-1} \sum_{h=1}^{\infty} \pi(h) p_{h 0}$

which is always a probability distribution on $\{j\}$. The corresponding conditional mean converges to

$$
\sum_{j=1}^{\infty} j \pi(j) \sum_{h=1}^{\infty} p_{j h}^{(k-1)} p_{h 0} / \gamma^{k-1} \sum_{h=1}^{\infty} \pi(h) p_{h 0}<+\infty .
$$

In fact

$$
P\left[Z_{n}=j \mid T=n+k\right]=\sum_{i=1}^{\infty} \lambda_{i} p_{i j}^{(n)} \sum_{h=1}^{\infty} p_{j h}^{(k-1)} p_{h 0} / \sum_{h=1}^{\infty} \sum_{i=1}^{\infty} \lambda_{i} p_{i h}^{(n+k-1)} p_{h 0}
$$

where $\sum_{h=1}^{\infty} p_{j h}^{(\boldsymbol{k}-1)} p_{h 0}=p_{j 0}^{(\boldsymbol{k})}-p_{j 0}^{(\boldsymbol{k}-1)}=F_{k}(0)^{j}-F_{k-1}(0)^{j}$. We proceed as in (I) and (II). Note that $p_{h 0}=\left(p_{10}\right)^{h}$. The special case $m=1, k=0$, $\lambda_{1}=1$ in (17) is the union of Theorems 1 and 2 in [10].

(IV). Letting $k \rightarrow+\infty$ in (15), (16), (17) and (18) we find the following limits:

In (15), if $m \neq 1$,

$$
\frac{\pi(j)}{\sum_{h=1}^{\infty} \pi(h) q^{h}} \lim _{k \rightarrow \infty} \frac{p_{1 r}^{(k)}}{\gamma^{k}} \sum_{h=1}^{\infty} \frac{p_{j h}^{(k)}}{p_{1 r}^{(k)}} q^{h}=A^{\prime}(q)^{-1} j q^{j-1} \pi(j)
$$

by (11) and (13). This is positive if and only if the process is $R$-positive, in which case it agrees with the result in [11].

Similarly in (16), if the process is $R$-positive the limit is $A^{\prime}(q)^{-1}$ $\cdot \sum_{j=1}^{\infty} j^{2} q^{j-1} \pi(j)$. In (17) the limit is $A^{\prime}(q)^{-1} j q^{j-1} \pi(j)$.

In (18) if the process is $R$-positive then we obtain $A^{\prime}(q)^{-1}$ - $\sum_{j=1}^{\infty} j^{2} q^{j-1} \pi(j)$. If the process is not $R$-positive the limit is $+\infty$. In fact, with $x_{0}=F(0)$, (18) becomes

$$
\begin{array}{r}
\frac{\sum_{j=1}^{\infty} j \pi(j)\left[F_{k}(0)^{j}-F_{k-1}(0)^{j}\right]}{\gamma^{k-1} A\left(x_{0}\right)}=\frac{A^{\prime}\left(F_{k}(0)\right) F_{k}(0)-A^{\prime}\left(F_{k-1}(0)\right) F_{k-1}(0)}{\gamma^{k-1} A\left(x_{0}\right)} \\
\geqq\left[\frac{A^{\prime}\left(F_{k-1}\left(x_{0}\right)\right)}{\gamma^{k-1}}-\frac{1}{\gamma} \frac{A^{\prime}\left(F_{k-2}\left(x_{0}\right)\right)}{\gamma^{k-2}}\right] \cdot \frac{F_{k-1}(0)}{A\left(x_{0}\right)} .
\end{array}
$$


Using (8) and the equality $F_{k-1}^{\prime}\left(x_{0}\right)=F^{\prime}\left(F_{k-2}\left(x_{0}\right)\right) F_{k-2}^{\prime}\left(x_{0}\right)$ we reduce the last expression to

$$
\frac{\gamma-F^{\prime}\left(F_{k-2}\left(x_{0}\right)\right)}{F_{k-2}^{\prime}\left(x_{0}\right)} \cdot \frac{A^{\prime}\left(x_{0}\right) F_{k-1}(0)}{\gamma F^{\prime}\left(F_{k-2}\left(x_{0}\right)\right) A\left(x_{0}\right)}
$$

and this converges to $+\infty$ because by the mean-value theorem

$$
\begin{aligned}
\frac{\gamma-F^{\prime}\left(F_{k-2}\left(x_{0}\right)\right)}{F_{k-2}^{\prime}\left(x_{0}\right)} & =\frac{F^{\prime}(1)-F^{\prime}\left(F_{k-2}\left(x_{0}\right)\right)}{F_{k-2}^{\prime}\left(x_{0}\right)} \geqq \frac{F^{\prime \prime}\left(F_{k-2}\left(x_{0}\right)\right)\left(1-F_{k-2}\left(x_{0}\right)\right)}{F_{k-2}^{\prime}\left(x_{0}\right)} \\
& \geqq F^{\prime \prime}\left(F_{k-2}\left(x_{0}\right)\right)\left(1-x_{0}\right) \quad \text { and } \quad F^{\prime \prime}(1)=+\infty
\end{aligned}
$$

(V). Let us now assume $P\left[Z_{0}=1\right]=1$. E. Seneta and D. Vere-Jones [11] considered the expected proportion of time spent in the state $j$ up to time $n$, under the condition $n<T<+\infty$ :

$$
\tau_{j}(n)=\frac{1}{n} \sum_{\nu=1}^{n}\left(p_{1 j}^{(\nu)} \sum_{k=1}^{\infty} p_{j k}^{(n-\nu)} q^{k}\right) / \sum_{k=1}^{\infty} p_{1 k}^{(n)} q^{k} .
$$

If the process is $R$-positive this converges to $A^{\prime}(q)^{-1} j q^{j-1} \pi(j)$ [11]. We shall prove that if the process is not $R$-positive then $\lim _{n \rightarrow \infty} \tau_{j}(n)$ $=0, j=1,2, \cdots$. Let $m<1$ and note that for fixed $N$

$$
\begin{aligned}
\lim _{n \rightarrow \infty} \frac{p_{1 r}^{(n)}}{F_{n}^{\prime}\left(F_{N}(0)\right)} & =\lim _{n \rightarrow \infty} \frac{\frac{1}{r !} F^{(r)}(0) F^{\prime}(F(0)) \cdots F^{\prime}\left(F_{n-1}(0)\right)}{F^{\prime}\left(F_{N}(0)\right) F^{\prime}\left(F_{N+1}(0)\right) \cdots F^{\prime}\left(F_{n+N-1}(0)\right)} \\
& =\lim _{n \rightarrow \infty} \frac{\frac{1}{r !} F^{(r)}(0) F^{\prime}(F(0)) \cdots F^{\prime}\left(F_{N-1}(0)\right)}{F^{\prime}\left(F_{n}(0)\right) \cdots F^{\prime}\left(F_{n+N-1}(0)\right)}=\frac{p_{1 r}^{(N)}}{\gamma^{N}} .
\end{aligned}
$$

Note also that when $n>\nu$

$$
\begin{aligned}
p_{1 r}^{(n)} & =\frac{1}{r !} F^{(r)}(0) F^{\prime}(F(0)) \cdots F^{\prime}\left(F_{n-1}(0)\right) \\
& =p_{1 r}^{(\nu)} F^{\prime}\left(F_{\nu}(0)\right) F^{\prime}\left(F_{\nu+1}(0)\right) \cdots F^{\prime}\left(F_{n-1}(0)\right)=p_{1 r}^{(v)} F_{n-\nu}^{\prime}\left(F_{\nu}(0)\right) .
\end{aligned}
$$

Thus when $N$ is fixed and $n>N$ we have

$$
\tau_{j}(n)=\frac{1}{n} \sum_{\nu=1}^{n} \frac{p_{i j}^{(\nu)}}{p_{1 r}^{(v)}}\left(\frac{p_{1 r}^{(n-\nu)}}{F_{n-\nu}^{\prime}\left(F_{\nu}(0)\right)} \sum_{k=1}^{\infty} \frac{p_{j k}^{(n-\nu)}}{p_{1 r}^{(n-\eta}}\right) / \sum_{k=1}^{\infty} \frac{p_{1 k}^{(n)}}{p_{1 r}^{(n)}} .
$$

The denominator converges to $\sum_{k=1}^{\infty} \pi(k)$. For $\nu>N$ we have $F_{n-\nu}^{\prime}\left(F_{\nu}(0)\right) \geqq F_{n-\nu}^{\prime}\left(F_{N}(0)\right)$ so that the numerator is 


$$
\leqq \frac{1}{n} \sum_{\nu=1}^{N}(\cdots)+\frac{1}{n} \sum_{\nu=N+1}^{n} \frac{p_{1 j}^{(\nu)}}{p_{1 r}^{(v)}}\left(\frac{p_{1 r}^{(n-\nu)}}{F_{n-\nu}^{\prime}\left(F_{N}(0)\right)} \sum_{k=1}^{\infty} \frac{p_{j k}^{(n-\nu)}}{p_{1 r}^{(n-\nu)}}\right) .
$$

By (13), (19) and the abelian lemma [11, p. 409] the lim $\sup _{n \rightarrow \infty}$ of the numerator is $\leqq \pi(j)\left(p_{1 r}^{(N)} / \gamma^{N}\right) j \sum_{k=1}^{\infty} \pi(k)$ and this holds for every $N$.

If $m=1$ one must write

$$
\begin{aligned}
\tau_{j}(n) \leqq & \frac{\sum_{k=1}^{\infty} p_{j k}^{(n)}}{\sum_{k=1}^{\infty} p_{1 k}^{(n)}} \cdot \frac{p_{1 r}^{(n)}}{F_{n}{ }^{\prime}\left(F_{N}(0)\right)} \cdot \frac{\frac{1}{n} \sum_{\nu=0}^{n-1} a_{N}(\nu)}{a_{N}(n)} \\
& \frac{\sum_{\nu=1}^{N}\{\cdots\}+\sum_{\nu=N+1}^{n} \frac{p_{1 j}^{(\nu)}}{p_{1 r}^{(v)}} a_{N}(n-\nu)}{\sum_{\nu=0}^{n-1} a_{N}(\nu)}
\end{aligned}
$$

where

$$
a_{N}(n)=\frac{p_{1 r}^{(n)}}{F_{n}^{\prime}\left(F_{N}(0)\right)} \sum_{k=1}^{\infty} \frac{p_{j k}^{(n)}}{p_{1 r}^{(n)}}
$$

and apply Lemma $\mathrm{A}$ in $[1$, p. 20].

The quasi-mixing consequences of (4) in the irreducible case will be indicated elsewhere (cf. [9] for the stochastic case). In concluding the author wishes to express his indebtedness to E. Seneta and D. Vere-Jones for the privilege of reading preprints of some of their papers.

AdDED in PROOF. In connection with the lemma, G. E. H. Reuter who read a preprint of the present note has observed that the sequence $p_{1 j}^{(n)} /\left(p_{10}^{(n)}-p_{10}^{(n-1)}\right)=F_{n}^{(j)}(0) / j !\left(F_{n}(0)-F_{n-1}(0)\right), \quad n=1,2, \cdots$ is also increasing (use (3) and the mean value theorem) and can be used interchangeably with $p_{1 j}^{(n)} / p_{1 r}^{(n)}$.

\section{REFERENCES}

1. K. L. Chung, Markov chains with stationary transition probabilities, 1st ed., Springer-Verlag, Berlin, 1960.

2. W. Feller, An introduction to probability theory and its applications, Vol. 1, 2nd ed., Wiley, New York, 1957.

3. T. E. Harris, Branching processes, Springer-Verlag, Berlin, 1963.

4. C. R. Heathcote, E. Seneta and D. Vere-Jones, A refinement of two theorems in the theory of branching processes, Teor. Verojatnost. i Primenen. 12 (1967), 341-346. 
5. A. Joffe, On the Galton-Watson branching process with mean less than one, Ann. Math. Statist. 38 (1967), 264-266.

6. S. Karlin and J. McGregor, Spectral theory of branching processes. I, Z. Wahrscheinlichkeitstheorie und Verw. Gebiete 5 (1966), 6-33.

7. H. Kesten, P. Ney and F. Spitzer, The Galton-Watson process with mean one and finite variance, Teor. Verojatnost. i Primenen. 11 (1966), 579-611.

8. A. N. Kolmogorov, Zur Lösung einer biologischen Aufgabe, Izv. Naučn.-Issled. Inst. Mat. i Meh. Tomsk. Gosudarstv. Univ. 2 (1938), 1-6.

9. F. Papangelou, Strong ratio limits, R-recurrence and mixing properties of discrete parameter Markov processes, Z. Wahrscheinlichkeitstheorie und Verw. Gebiete 8 (1967), 259-297.

10. E. Seneta, The Galton-Watson process with mean one, J. Appl. Probability 4 (1967), 489-495.

11. E. Seneta and D. Vere-Jones, On quasi-stationary distributions in discrete-time Markov chains with a denumerable infinity of states, J. Appl. Probability 3 (1966), 403-434.

12. D. Vere-Jones, Ergodic properties of non-negative matrices. I, Pacific J. Math. 22 (1967), 361-386.

13. A. M. Yaglom, Certain limit theoerms of the theory of branching random processes, Dokl. Akad. Nauk SSSR 56 (1947), 795-798. (Russian)

UNIVERSITY OF HEIDELBERG 